Global and local gyrokinetic simulations of high-performance discharges in view of ITER

This article has been downloaded from IOPscience. Please scroll down to see the full text article.

2013 Nucl. Fusion 53073003

(http://iopscience.iop.org/0029-5515/53/7/073003)

View the table of contents for this issue, or go to the journal homepage for more

Download details:

IP Address: 130.183.100.98

The article was downloaded on 07/08/2013 at 12:41

Please note that terms and conditions apply. 


\title{
Global and local gyrokinetic simulations of high-performance discharges in view of ITER
}

\author{
F. Jenko ${ }^{1}$, D. Told ${ }^{1}$, T. Görler ${ }^{1}$, J. Citrin ${ }^{2}$, A. Bañón Navarro ${ }^{1}$, \\ C. Bourdelle ${ }^{3}$, S. Brunner ${ }^{4}$, G. Conway ${ }^{1}$, T. Dannert ${ }^{5}$, H. Doerk ${ }^{1}$, \\ D.R. Hatch ${ }^{1}$, J.W. Haverkort ${ }^{2}$, J. Hobirk ${ }^{1}$, G.M.D. Hogeweij ${ }^{2}$, \\ P. Mantica ${ }^{6}$, M.J. Pueschel ${ }^{7}$, O. Sauter ${ }^{4}$, L. Villard ${ }^{4}$, E. Wolfrum ${ }^{1}$ \\ and the ASDEX Upgrade Team
}

\author{
${ }^{1}$ Max-Planck-Institut für Plasmaphysik, EURATOM-Assoziation, Boltzmannstr. 2, 85748 \\ Garching, Deutschland \\ ${ }^{2}$ FOM Institute DIFFER, Association EURATOM-FOM, 3430 Nieuwegein, The Netherlands \\ ${ }^{3}$ CEA, IRFM, F-13108 Saint Paul Lez Durance, France \\ ${ }^{4}$ École Polytechnique Fédérale de Lausanne (EPFL), EURATOM Association, 1015 \\ Lausanne, Switzerland \\ ${ }^{5}$ Rechenzentrum Garching, Boltzmannstr. 2, 85748 Garching, Germany \\ ${ }^{6}$ Istituto di Fisica del Plasma 'P. Cadirola', Associazione EURATOM-ENEA-CNR, \\ Milano, Italy \\ ${ }^{7}$ Department of Physics, University of Wisconsin-Madison, Madison, WI 53706, USA \\ E-mail: frank.jenko@ipp.mpg.de
}

Received 2 January 2013, accepted for publication 3 May 2013

Published 23 May 2013

Online at stacks.iop.org/NF/53/073003

\begin{abstract}
One of the key challenges for plasma theory and simulation in view of ITER is to enhance the understanding and predictive capability concerning high-performance discharges. This involves, in particular, questions about high- $\beta$ operation, ion temperature profile stiffness, and the physics of transport barriers. The goal of this contribution is to shed light on these issues by means of physically comprehensive $a b$ initio simulations with the global gyrokinetic code GENE, applied to discharges in TCV, ASDEX Upgrade, and JET-with direct relevance to ITER.
\end{abstract}

(Some figures may appear in colour only in the online journal)

\section{Introduction}

The ultimate goal of magnetic confinement fusion theory is to provide the necessary physics understanding and predictive capability for developing efficient fusion devices. This involves, very importantly, addressing the cross-field turbulent transport of energy, particles, and momentum which determines the quality of magnetic confinement [1]. Given the enormous complexity of the problem, a main challenge is thus to develop and validate $a b$ initio simulation tools based on the gyrokinetic theory $[2,3]$. One of these tools is the GENE code [4-6] which can treat an arbitrary number of ion species, fully kinetic electrons, electromagnetic effects, inter- and intra-species collisions, as well as fluctuations on sub-ion-gyroradius scales. Moreover, GENE can be run in a radially global (full-torus/annulus/wedge), flux-surface global (non-spectral in the binormal direction), or local (flux-tube) mode, either gradient- or flux-driven, and it is interfaced with various MHD equilibrium codes and transport solvers. Most of these features are used in the studies presented in this work.

Some of the outstanding open questions regarding the successful operation of ITER are related to the nature of high-performance discharges. This involves issues linked to the scaling of core anomalous transport with $\rho^{*}$ and $\beta$, the role of profile stiffness, as well as the physics of edge and internal transport barriers. In this paper, we would like to shed some light on these issues by means of physically comprehensive GENE simulations applied to discharges in TCV, ASDEX Upgrade, and JET-with direct relevance to ITER. The rest of the paper is structured as follows: in section 2, finite-size effects are studied by means of highrealism simulations, comparing local and global simulations for specific ASDEX Upgrade and JET discharges. It will be shown that the local approximation works very well for such medium-scale to large-scale devices, justifying the use of coupled (via a transport solver) flux-tube runs for the 
(a) ASDEX Upgrade

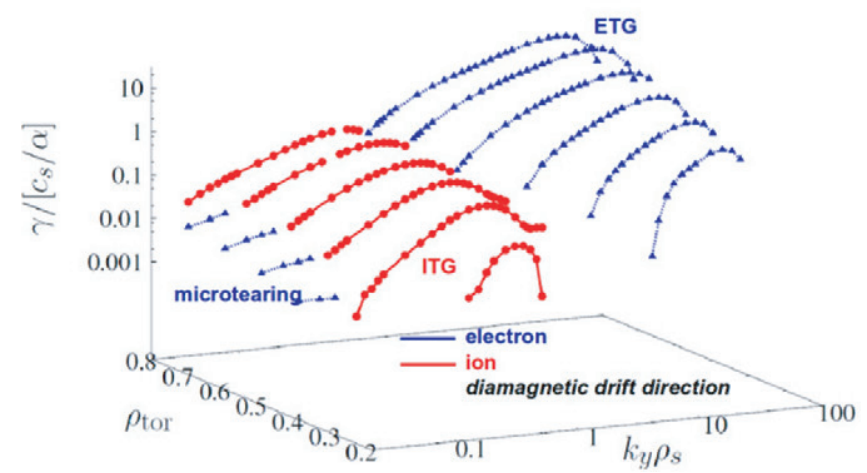

(b) JET

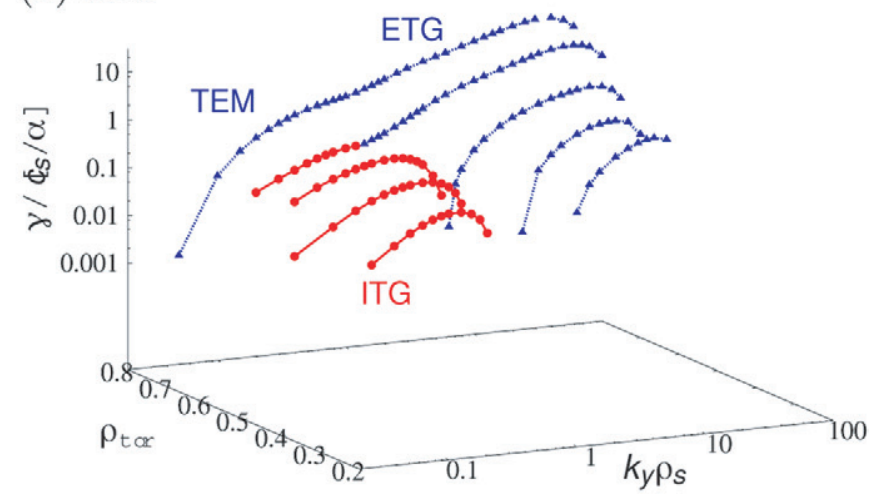

Figure 1. Normalized linear growth rates for (a) ASDEX Upgrade discharge \#22009 $(t=4.1 \mathrm{~s})$ and $(b)$ JET discharge \#70084 $(t=50 \mathrm{~s})$ as functions of the flux-surface label $\rho_{\text {tor }}$ and the binormal wave number $k_{\mathrm{y}}$. Blue, filled triangles indicate modes which move in the electron diamagnetic drift direction while red, filled circles indicate modes propagating in the ion diamagnetic drift direction.

core region of ITER-like devices in standard scenarios. In section 3 , some key questions regarding high- $\beta$ operation will be addressed, summarizing a large body of recent work in that area. In this context, it will be discussed that linearly unstable or stable microtearing modes (MTMs) tend to produce significant levels of magnetic electron heat transport, while-at least in ion temperature gradient (ITG)-driven turbulence- the electrostatic fluxes decrease with increasing $\beta$. In section 4 , the threshold and stiffness of ITG turbulence is studied, in particular in relation to magnetic shear and toroidal rotation. The simulations are then compared directly with some recent dedicated JET experiments. Finally, in section 5, the role and nature of the residual turbulent transport in electron internal transport barriers is discussed, focusing on discharges from the TCV experiment. It is shown that electron temperature gradient (ETG)-driven turbulence may be expected to set a lower limit in this type of barrier, highlighting the multi-scale nature of barrier physics and the need to incorporate sub-iongyroradius scales into corresponding modelling efforts. A brief summary will be given in section 6 .

\section{Finite-size effects in high-realism simulations}

In this section, we will study finite-size effects by means of high-realism simulations, comparing local and global simulations for specific ASDEX Upgrade and JET discharges. These investigations touch upon the important issue of the $\rho^{*}$ scaling of turbulent transport. As it turns out, the local approximation works very well for such medium-scale to largescale devices, motivating the use of coupled (via a transport solver) flux-tube runs for the core region of ITER-like devices in standard scenarios.

\subsection{Two L-mode discharges}

First, we would like to investigate the role of finite-size effects by means of high-realism simulations, comparing local and global simulations for specific ASDEX Upgrade and JET discharges. In this context, we will address the question to which degree global and local simulations differ for mediumscale or large-scale devices, building on previous studies which usually employed approximations such as adiabatic electrons and/or circular flux surfaces (see, e.g., [7-9]). At the same time, comparisons with experimental transport levels are performed, assessing the predictive capability of the present numerical setup.

Two L-mode discharges have been selected for this study, leaving high-performance $\mathrm{H}$-mode discharges for future comparisons. The first one is the ASDEX Upgrade discharge \#22009 (with 2.9 MW of NBI heating) during the L-mode phase at $t=4.1 \mathrm{~s}$. Both the MHD equilibrium and the plasma profiles are taken directly from the experimental database. The only exception is a mild adaptation of the ion temperature profile which is taken to be equal to the electron temperature in the outer core plasma (at $\rho_{\text {tor }}>0.6$ ) where only very a few data points exist. The second one is the JET discharge \#70084 (with 4.9 MW of ICRH heating and 0.6 MW of ohmic heating), also an L-mode plasma. Again, both the MHD equilibrium and the plasma profiles are taken directly from the experimental database. Given the similarity of both of these machines to ITER, such an investigation may be considered as a reasonable basis for an extrapolation to ITER.

\subsection{Linear stability studies}

A preliminary picture of the kind of turbulence to be expected can be obtained via local, linear GENE runs, identifying the existing microinstabilities. The simulations retain perpendicular magnetic perturbations, collisions (both energy and pitch-angle scattering) and realistic magnetic geometry and are run at zero equilibrium $E \times B$ flow shear. Some representative simulation results are displayed in figure 1. Here, $\gamma$ and $k_{\mathrm{y}}$ denote the linear growth rate and binormal wavenumber, respectively; $\rho_{\mathrm{s}}$ is the ion sound radius (ion gyroradius at electron temperature), $c_{\mathrm{s}}=\rho_{\mathrm{s}} \Omega_{\mathrm{i}}$ is the ion sound speed, and $\alpha=\sqrt{\Psi_{\text {tor }} /\left(\pi B_{\text {axis }}\right)}$ is an effective minor radius, defined in terms of the toroidal magnetic flux $\Psi_{\text {tor }}$ and the magnetic field $B_{\text {axis }}$ on the magnetic axis. In both cases, ITG-driven modes tend to play an important role on ion-gyroradius scales, while there are also ETGdriven modes on electron-gyroradius scales. In addition, the ASDEX Upgrade discharge exhibits linearly unstable MTMs at very low wavenumbers, while the JET discharge is dominated by trapped electron modes (TEMs) at the outermost radial position. In principle, it would be desirable to perform multi-scale nonlinear simulations covering the 


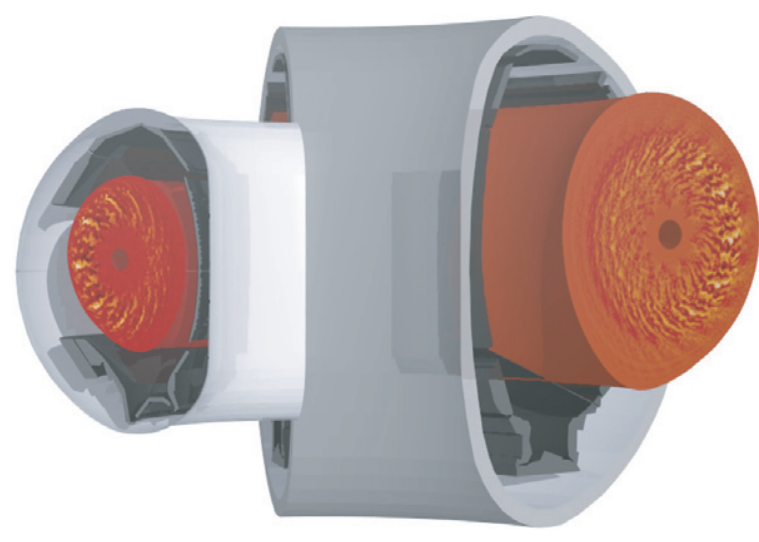

Figure 2. Ion temperature fluctuations in toroidal fractions of the computational domains for the nonlinear ASDEX Upgrade (left) and JET (right) simulations in comparison with the vessels.

entire flux surface while resolving electron-gyroradius scales in order to get a comprehensive overall view of the different transport mechanisms at work, but such simulations are not yet feasible- even on the largest present-day supercomputers. Thus, we will focus on ion-gyroradius scales, neglecting a possible role of ETG modes and MTMs. While the contributions to the electron heat flux from these modes are neglected this way although they might be significant, the ion heat and particle fluxes should be captured reasonably well (assuming limited nonlinear cross-mode couplings).

\subsection{Nonlinear simulations on ion-gyroradius scales}

For the nonlinear global GENE simulations, the numerical resolution in the cross-field directions for the ASDEX Upgrade (JET) simulations is chosen to be $k_{\mathrm{y}}^{\max } \rho_{\mathrm{s}} \sim 1.7(1.9)$ and $\Delta x \sim$ $0.5(0.65) \rho_{\mathrm{s}}$. Here, $\rho_{\mathrm{s}}$ is taken at the reference flux surface $\left(\rho_{\text {tor }}=0.5\right)$. The minimum toroidal mode number is 5(8), and the radial domain covers about $80 \%$ of the minor radius (see figure 2). The numerical resolution in the field-line-following direction is $24(32)$ grid points, while $96 \times 32(96 \times 64)$ grid points are employed for the parallel velocity and magnetic moment directions. In the toroidal direction, 32(48) modes are kept, while the radial direction is discretized using 512(1024) grid points. The increase in the number of grid points from ASDEX Upgrade to JET is due to the significantly larger machine size $-\rho^{*}=\rho_{\mathrm{s}} / \alpha=1 / 365$ versus $\rho^{*}=1 / 674-$ and stronger profile variations. As the local simulations, the global runs retain perpendicular magnetic fluctuations, collisions and realistic magnetic geometry, while no external momentum input is included. In all of these simulations, a perpendicular hyperdiffusion model has been used in order to avoid unphysical spectral pile-ups caused by neglecting smallscale (ETG-driven) dynamics.

Despite these limitations, one finds fairly good agreement between the total (i.e. summed over species and electrostatic/electromagnetic contributions) theoretical and experimental heat flux levels, as can be seen in figure 3 . Here, the total electron/ion heat flux across a given flux surface is plotted versus the minor radius together with total injected power represented by a dashed line. It should be pointed out in this context that for small radii $\left(\rho_{\text {tor }}<0.3\right)$, the low fluxes are merely a reflection of the relatively extended radial heating profiles and thus physical. Meanwhile, the drops at large radii $\left(\rho_{\text {tor }}>0.85\right)$ are due to a dissipational buffer zone close to the outer boundary and thus unphysical. In the JET case, the strongly reduced transport in the outer core region $\left(\rho_{\text {tor }} \sim 0.8\right)$ is most likely related to the uncertainty of the ion temperature profile at the edge, which starts to depart significantly from the electron temperature profile outside of $\rho_{\text {tor }}>0.65$, despite the increasing collisionality. In addition, there could be further contributions via the electron heat transport channel from the unresolved sub-iongyroradius scale turbulence, as indicated by the linear results, see figure $1(b)$. Other possible explanations include nonlocal effects involving the edge region, with strong turbulence propagating radially inwards - although there is no numerical evidence of such dynamics up to now. Even more importantly, figure 3 shows that the global and local simulation results agree fairly well, especially when taking into account the significant error bars of the quasi-stationary transport levels. Meanwhile, in a similar study for the TCV tokamak (not shown here), strong deviations have been observed. In that case, one had $\rho^{*}>1 / 100$. Consequently, for machine sizes somewhere between TCV and ASDEX Upgrade, there tends to be a transition from nonlocal to local behaviour, in line with previous studies with reduced physics [7-9]. From this, one may conclude that any deviations from gyroBohm scaling in ITER-like machines will probably have to come from edge effects. Moreover, this finding confirms the notion that in the absence of internal transport barriers, plasma turbulence in the core of large devices can be described by coupled fluxtube runs, an approach which considerably reduces the total computational effort (see, e.g., [10]).

\section{Anomalous transport in high- $\beta$ plasmas}

In addition to the $\rho^{*}$ scaling of turbulent transport, its dependence on $\beta$ is another question of vital interest for future fusion experiments. In order to maximize the fusion power (and bootstrap current fraction), one will want ITER or other future burning plasma devices to operate at high $\beta$. Therefore, a thorough understanding of finite- $\beta$ effects in turbulent transport is essential for achieving a predictive capability. Unfortunately, experimental studies yield scalings of confinement with $\beta$ which vary widely. While both ASDEX Upgrade and JT-60U exhibit unfavourable $\beta$ scalings [11], other devices point to a rather weak $\beta$ dependence $[12,13]$. This situation sparked intense theoretical and computational efforts over the last few years, examining the mechanisms by which magnetic stochasticity and electromagnetic transport arise in turbulent systems driven by ITG modes or TEMs. In addition, there have been some initial studies regarding the role of microtearing turbulence in standard tokamaks at sufficiently high- $\beta$ values. These results have been presented in separate papers, and we will only try to summarize some key findings here, referring the interested reader to the respective references for more detailed accounts.

\subsection{High- $\beta$ ITG turbulence and nonlinearly driven MTMs}

Over the last years, several gyrokinetic studies have explored electromagnetic effects in ITG turbulence, based 


\section{(a) ASDEX-Upgrade}

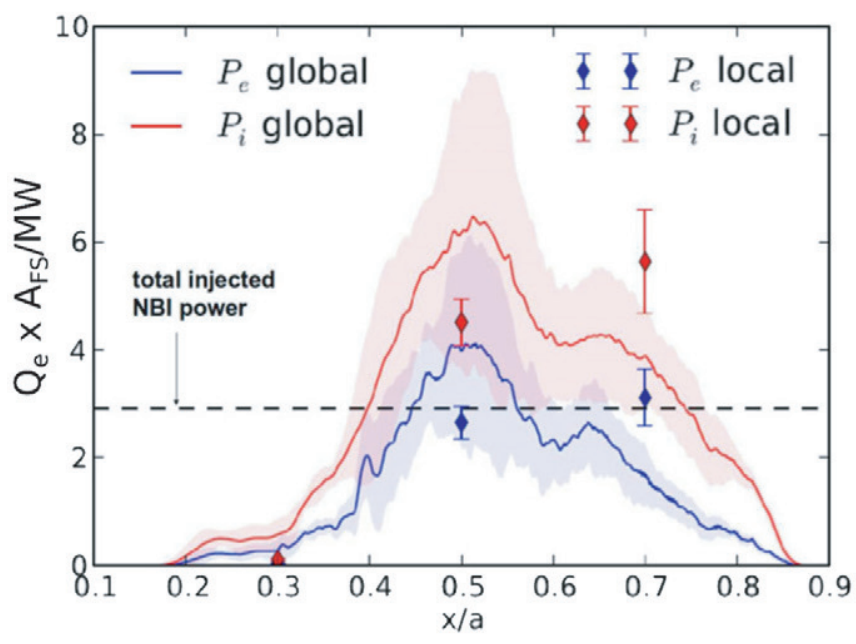

(b) JET

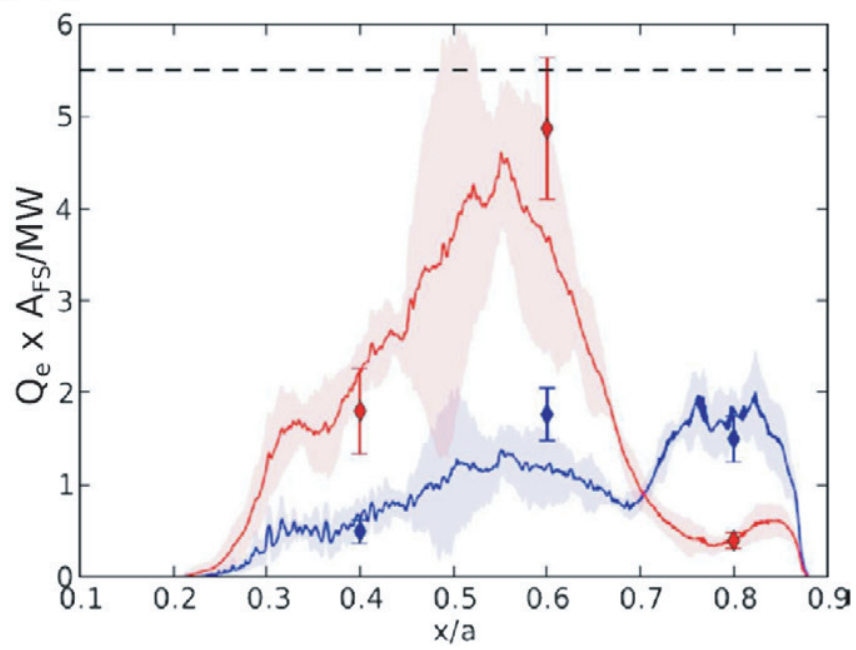

Figure 3. Global and local heat transport simulation results for two different discharges/machines demonstrating a reasonable agreement with the actual experimental power deposition and between the global and local code results. The shaded regions indicate the error bars of the global transport profiles, the dashed lines the total injected power.

on variations of the so-called Cyclone base case (CBC) [14] parameters [15-22]. These studies have shown that the electrostatic transport level decreases with increasing $\beta_{\mathrm{e}}$ (the ratio of electron pressure and magnetic pressure), holding all other dimensionless simulation parameters (like the normalized profile gradients) fixed. This behaviour is a reflection of the linear growth rates' $\beta_{\mathrm{e}}$ dependence, although it is nonlinearly enhanced via an increased zonal flow activity at high $\beta_{\mathrm{e}}$ [17]. Meanwhile, the electromagnetic transport level due to electrons following radially fluctuating magnetic field lines, at moderate to high $\beta_{\mathrm{e}}$ may become comparable to the electrostatic transport channels dominating at low $\beta_{\mathrm{e}}$. Interestingly, this behaviour contrasts sharply with the quasilinear expectations derived from the linear ITG mode: the latter induces an inward (up-gradient) electromagnetic electron heat flux which scales like $\beta_{\mathrm{e}}$, while an outward (downgradient) $\beta_{\mathrm{e}}^{2}$ scaling is observed in the nonlinear simulations. Moreover, it was found that even at very low $\beta_{\mathrm{e}}$ values, one obtains magnetic stochasticity, despite the fact that the ITG mode is characterized by ballooning (not tearing) parity, and is thus inefficient for breaking magnetic field lines [19,20]. Both these apparent contradictions could be resolved recently by demonstrating that the magnetic stochasticity and associated transport are not caused directly by the ITG mode. Rather, the salient mechanism is linearly stable MTMs (see, e.g. the references in [23]), which are driven nonlinearly and operate at the same perpendicular scales as the ITG modes [21]. These results offer a paradigm for electromagnetic transport which can be explored more extensively throughout parameter space in future studies.

\subsection{Role of linearly unstable MTMs}

In addition to these effects caused by linearly stable MTMs, there also tend to be linearly unstable MTMs in the outer core regions of standard tokamaks as has been shown in a series of linear gyrokinetic studies over the last few years [24-29]. Unstable MTMs-small-scale variants of MHD tearing modes-are electromagnetic (they exhibit a $\beta_{\mathrm{e}}$ threshold and intrinsically depend on magnetic fluctuations) modes which are driven primarily by ETGs. It was found in gyrokinetic simulations that MTMs are able to nonlinearly produce experimentally relevant heat flux levels [27, 28]. The spectral transfer of free energy could also be unraveled in this context. However, at present, there remain significant gaps in our understanding of MTMs, even linearly. Thus, much work still needs to be done in order to clearly grasp the drive mechanisms in various regimes, the dependence of the linear growth rate on various plasma parameters, as well as the nonlinear interaction between MTMs and other (coexisting) microinstabilities such as the ITG modes or TEMs. Such investigations are currently underway, but extremely challenging from a computational point of view. According to the results obtained thus far, it seems likely that MTMs are yet another relevant transport mechanism in standard tokamaks such as JET or ITER, in particular in high- $\beta$ discharges.

\subsection{Comparisons between simulation and experiment}

Direct comparisons between simulations and dedicated $\beta$ scaling experiments are another target to be achieved in the near future. Up to this point, simulations have always been performed in a way such that all normalized plasma parameters except for $\beta$ were held fixed. This does not reflect the situation in actual experimental $\beta$ variations. Instead of keeping the gradients constant, one should rather determine the change in gradients which is required to reproduce the fluxes correctly. In fact, all numerical input parameters should be adapted to each experimental situation, since there are likely to be nonlinear interaction effects between various parameter variations. Having said that, it may be expected on the basis of existing simulation results that experiments operating sufficiently close to the ideal MHD ballooning limit should be subject to a gradual shift from purely electrostatic to mixed electrostatic/electromagnetic turbulent transport, catalysed by MTMs which are either linearly or nonlinearly driven. Under which circumstances and to which degree this effect is reflected in the overall $\beta$ scaling of the energy confinement time remains 

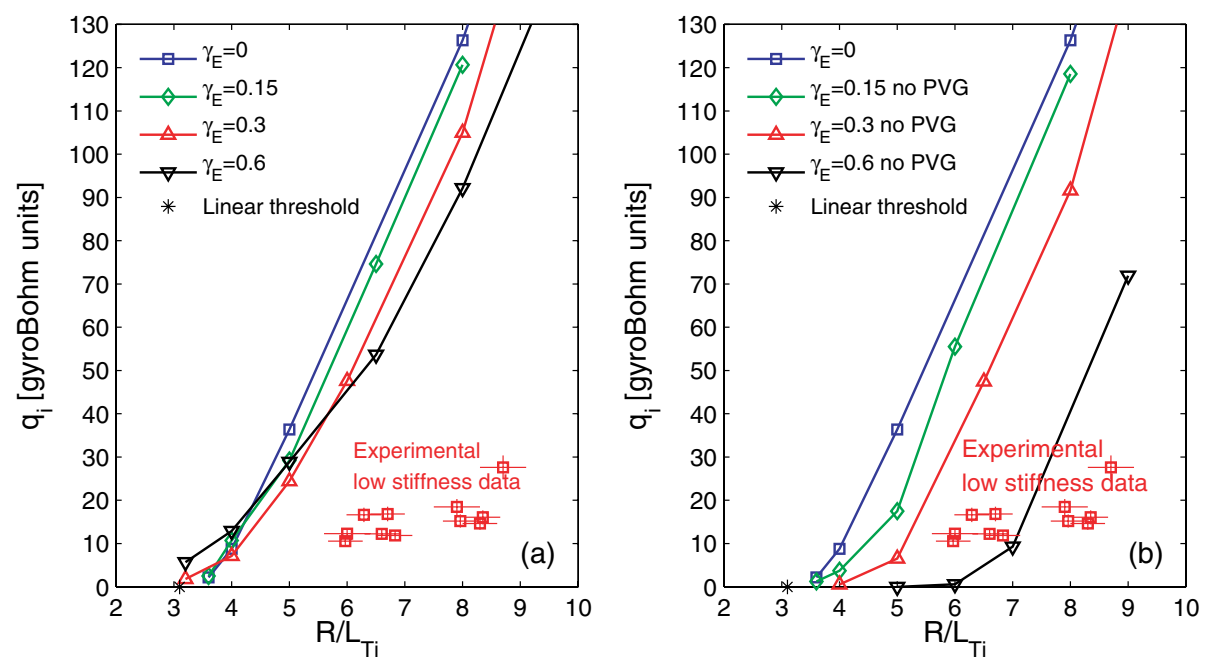

Figure 4. Nonlinear GENE $R / L_{T i}$ scans based on 70084 parameters at $\rho_{\text {tor }}=0.33$ and various levels of $\gamma_{E}\left[c_{\mathrm{s}} / R\right]$. Runs including PVG destabilization are seen in $(a)$. Runs ignoring PVG destabilization are seen in $(b)$. The results are compared with the low stiffness data at $\rho_{\text {tor }}=0.33$ from $[31]$.

to be investigated. An important open question in this context concerns the relative role of core and edge transport. Given our limited predictive capability regarding the latter, reliable predictions and simulation-experiment comparisons will have to be focused on core confinement in the foreseeable future. Nevertheless, the dependence of the quality of magnetic confinement on $\beta$ remains a crucial question to be addressed in light of ITER.

\section{Simulations of ion temperature profile stiffness in JET experiments}

Next, we will turn to the question of ion temperature profile threshold and stiffness. Given that future devices such as ITER will operate at high core temperatures, the normalized heat fluxes (in gyroBohm units) will be rather low, suggesting that the typical operational points will be close to the (nonlinear) threshold. This motivates a closer look at this particular physics question. Interestingly, we will find that finite $\beta$ effects play an important role in this context.

\subsection{Some recent JET results}

In recent JET experiments, a reduction of ion temperature profile stiffness at low normalized radii $\left(\rho_{\text {tor }}<0.5\right)$ has been observed [30,31]. This has been hypothesized to be related to the correlation between low magnetic shear $(\hat{s})$ and increased flow shear in the low stiffness discharges. A previous nonlinear gyrokinetic study based on such discharges, as detailed in [31], reported only an ITG threshold shift with rotation, as opposed to a decrease in stiffness as observed. We extend this previous work to investigate whether the stiffness reduction can be understood through gyrokinetic modelling.

Three JET discharges (\#70084, \#66130 and \#66404) from the dataset presented in [31] were analysed at $\rho_{\text {tor }}=0.33$. The first one, \#70084, corresponds to a representative low rotation, low-flux discharge. The latter, \#66130 and \#66404, are discharges which displayed significantly reduced stiffness in the ion heat channel. The differences in parameters apart from rotation within the chosen discharge set-such as $R / L_{n}$, $\beta_{\mathrm{e}}, Z_{\mathrm{eff}}$, and fast particle content-were closely investigated with interpretative CRONOS [32] simulations to see whether that could lead to an appreciable stiffness reduction in subsequent nonlinear simulations. The flow shear is represented by the $\gamma_{E}$ parameter. For purely toroidal rotation-justified by the strongly driven NBI discharges discussed-one has $\gamma_{E}=$ $-\frac{r}{q} \frac{\partial \Omega}{\partial r} \frac{R}{c_{\mathrm{s}}}$. For the low/high rotation discharges, $\gamma_{E}=0.1 / 0.3$ was assumed, which are representative values for the low and high stiffness discharges from the dataset in [31]. All simulations carried out were local, which is justified since $1 / \rho^{*} \sim 500$ for the range of plasma parameters studied here $[8,9]$.

From nonlinear simulations, it was found that the differences between the discharges in $R / L_{n}$, the effect of rotation on the MHD equilibria, fast particle dilution, and increased Shafranov shifts due to suprathermal pressure, did not have a significant impact on the stiffness for our parameters. Therefore, we focus in the following subsections on the impact of rotation and electromagnetic effects on the predicted stiffness levels. We then provide a full comparison between the experimental and nonlinearly predicted fluxes for the three discharges, where the $Z_{\text {eff }}$ sensitivity is also addressed.

\subsection{Stiffness sensitivity to rotation}

In this subsection we isolate the effect of rotation on stiffness. Collisionless, electrostatic simulations based on \#70084 parameters (with $\hat{s} / q=0.2 / 1.3$ ) are carried out, applying an analytical circular geometry [33]. The predicted gyroBohm normalized ion heat fluxes from the $R / L_{T i}$ scans are shown in figure 4 . The sensitivity to $\gamma_{E}$ is examined. Even for $\gamma_{E}=0.6$, double the highest level of flow shear achieved in the reference data set from [31], the simulated level of reduced stiffness is significantly less than the experimental observation, as seen by the direct comparison with the reference data. However, interesting effects related to the competition between stabilizing $E \times B$ shear and destabilizing parallel velocity gradient (PVG) modes-particularly in the vicinity of the threshold-are observed. At low $R / L_{T i}$, the PVG destabilization can dominate over the ITG turbulence, 

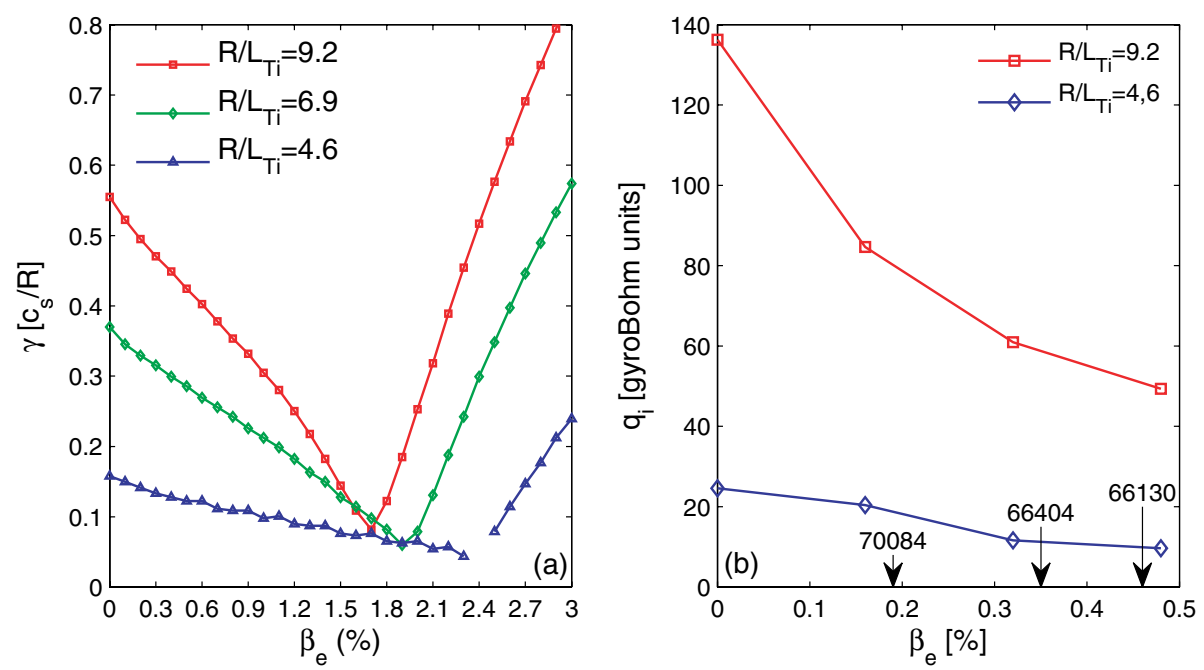

Figure 5. Linear $(a)$ and nonlinear $(b) \beta_{\mathrm{e}}$ scans with 66404 parameters at $\rho_{\text {tor }}=0.33 . R / L_{T i}$ and $\gamma_{E}$ are varied. Runs were with collisions and numerical geometry.

reducing stiffness in that region of parameter space. Due to the PVG destabilization, the fluxes do not continue to decrease towards the ITG instability thresholds. In figure $4(b)$ the parallel velocity gradients were artificially removed from the system, and the picture reverts to a threshold shift. However, regardless of the PVG assumption, the GENE simulations do not predict a significant reduction in stiffness due to flow shear.

\subsection{Impact of $\beta_{\mathrm{e}}$ on the stiffness level}

In this subsection the sensitivity of the stiffness on $\beta_{\mathrm{e}}$ is examined. The simulations carried out take discharge \#66404 parameters as a reference. Linear (at $k_{\mathrm{y}} \rho_{\mathrm{s}}=0.4$ ) and nonlinear $\beta_{\mathrm{e}}$ scans are shown in figure 5. From the linear scans, we can see the range of experimental $\beta_{\mathrm{e}}$ values $(<0.5 \%)$ are significantly below the kinetic ballooning mode (KBM) thresholds. Approaching the KBM threshold, $\beta_{\mathrm{e}}$ stabilizes the ITG mode [34], also in the nonlinear case [17].

An important observation-also seen previously in the GENE simulations [18] - is that the nonlinear $\beta_{\mathrm{e}}$ ITG stabilization significantly exceeds the linear stabilization. A decrease in ion heat flux by a factor of $65 \%$ is seen in figure $5(b)$ for the $\gamma_{E}=0, R / L_{T i}=9.2$ case between $\beta_{\mathrm{e}}=0-0.48 \%$. Simultaneously, while the ion heat flux is reduced by $\beta_{\mathrm{e}}$ in the $\gamma_{E}=0, R / L_{T i}=4.6$ case, it is not totally quenched. This is indicative of a decrease in stiffness as opposed to a threshold shift. The reduced stiffness discharges \#66130 and \#66404 have increased $\beta_{\mathrm{e}}$ compared with the low-flux \#70084 discharge, consistent with the observed stabilization. The precise $\beta_{\mathrm{e}}$ values are indicated in figure 5 .

We can thus conclude that electromagnetic effects play a significant role in stiffness reduction for our parameters, even at relatively low values of $\beta_{\mathrm{e}}$. While this stiffness reduction is not sufficient to fully explain the experimentally observed stiffness reduction, it is a factor which must be taken into consideration.

\subsection{Full simulations including all effects}

Simulations of all three discharges in the data set at $\rho_{\text {tor }}=$ 0.33 were performed. These simulations included rotation, numerical geometry, the effect of rotation on the MHD equilibrium (through an increased effective pressure due to the centrifugal force), experimental $R / L_{n}$, finite $\beta$, collisions, experimental $Z_{\mathrm{eff}}$, and experimental $T_{\mathrm{e}} / T_{\mathrm{i}}$. The sensitivity of the results to $Z_{\text {eff }}$ was examined. The numerical geometry was generated by the Grad-Shafranov solver in the CRONOS interpretative simulations, consistent with the experimental pressure profiles a prescribed current profile consistent with the $\hat{s} / q=0.2 / 1.3$ assumption at $\rho_{\text {tor }}=0.33$. The $\hat{s}$ and $q$ values at $\rho_{\text {tor }}=0.33$ for discharge \#66404 obtained from the interpretative CRONOS simulation were $\hat{s} / q=0.4 / 1.7$.

The comparison between the GENE nonlinear simulations and the experimental heat fluxes is shown in figure 6. In $(a)$, the GENE simulations with experimental $T_{\mathrm{e}} / T_{\mathrm{i}}$ values and $Z_{\text {eff }}=1.4, Z_{\text {eff }}=1.9$ and $Z_{\text {eff }}=2.4$ are shown. In $(b)$, simulations with $T_{\mathrm{e}} / T_{\mathrm{i}}=Z_{\mathrm{eff}}=1$ are shown. The simulated ion heat flux value for the low rotation, low-flux casedischarge \#70084 — does agree with the experiment. However, the predicted fluxes for the high rotation cases are in most cases significantly higher than the experimental observation, indicating the insufficiently reduced stiffness in the gyrokinetic simulations.

Increased agreement between the simulated and measured flux values can be obtained be increasing $Z_{\text {eff }}$ to values above the line-averaged bremsstrahlung value $\left(\left\langle Z_{\text {eff }}\right\rangle \approx 1.9\right.$ for all discharges), as seen in figure $6(a)$. However, this is in strong disagreement with the charge-exchange measurements which measure $Z_{\text {eff }} \approx 1.4$. In addition, for the low rotation case, values of $Z_{\text {eff }}$ significantly beyond the charge-exchange measured values lead to a prediction of full stabilization, in disagreement with observation. Thus, we deem it unlikely that the flux discrepancy could be due to $Z_{\text {eff }}$ uncertainties. The impact of increasing the $\hat{s} / q$ value from our optimistically assumed $\hat{s} / q=0.2 / 1.3$ to the modelled value of $\hat{s} / q=$ $0.4 / 1.7$ for discharge \#66404 can also be seen in figure $6(a)$. This leads to an approximate doubling of the predicted flux.

The predicted and experimental fluxes can also be reconciled by artificially increasing $\gamma_{E}$ beyond the measured value from the toroidal rotation, and simultaneously ignoring PVG stabilization. This is consistent with assuming nonnegligible poloidal rotation. The original assumption of 

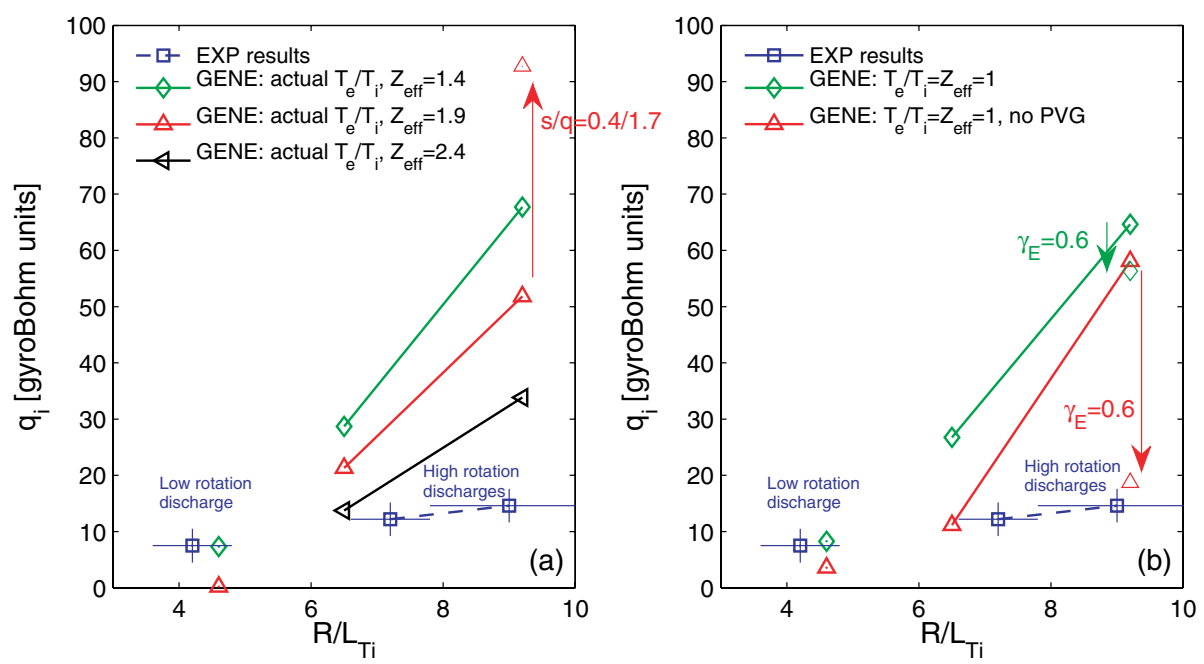

Figure 6. Comparison of nonlinear simulations and experimental results for the three separate discharges at $\rho=0.33$. The comparison with the experimental range of $Z_{\mathrm{eff}}$ and actual $T_{\mathrm{e}} / T_{\mathrm{i}}(1.08 / 1.25 / 1.14$ for $\# 70084 / \# 66130 / \# 66404$ respectively) is seen in $(a)$, while in $(b)$ the $T_{\mathrm{e}} / T_{\mathrm{i}}=Z_{\text {eff }}=1$ case is shown, both with and without the inclusion of PVG modes and for various values of $\gamma_{E}$. Runs were electromagnetic, with collisions, and with numerical geometry.

negligible poloidal rotation is justified by the NCLASS [35] neoclassical poloidal rotation predictions for the deuterium species within the CRONOs modelling, which are approximately an order of magnitude below the values necessary to provide significant turbulence suppression as observed. This motivates interest in measuring the poloidal rotation in the low-stiffnessregime discharges, to examine whether nonetheless any anomalous poloidal rotation is observed, potentially due to turbulent flow generation through a turbulent Reynolds stress.

In summary, our studies show that toroidal rotation alone (even in combination with low magnetic shear) cannot explain the ion temperature profile stiffness reduction observed in JET discharges. In addition, the usually assumed upshift of the critical gradient is counteracted (and often annihilated) by the PVG instability - an effect which is generally neglected in transport analyses. The key influence on stiffness reduction, according to our investigations, is due to finite $\beta_{\mathrm{e}}$ stabilization of ITG modes, in particular nonlinearly. By itself, it is still too small, however, to explain the experimental findings.

\section{Studies of transport barrier physics}

Finally, we would like to explore certain aspects of transport barrier physics. We are interested, most of all, in the sources of residual transport in established barriers. Here, a key question is to which degree ion-scale and electron-scale turbulence contribute to the overall fluxes. The answer dictates the range of spatio-temporal scales to be retained in future attempts to model the creation of transport barriers self-consistently.

\subsection{Internal transport barriers in $T C V$}

The global version of the GENE code is applied to discharges of the TCV tokamak exhibiting an electron internal transport barrier [36], retaining both gyrokinetic electrons and ions, and (for the linear simulations) also collisions and electromagnetic effects. The discharges studied here (first published in [37]) were fully non-inductive, but included small ohmic current perturbations to tweak the magnetic shear profile,

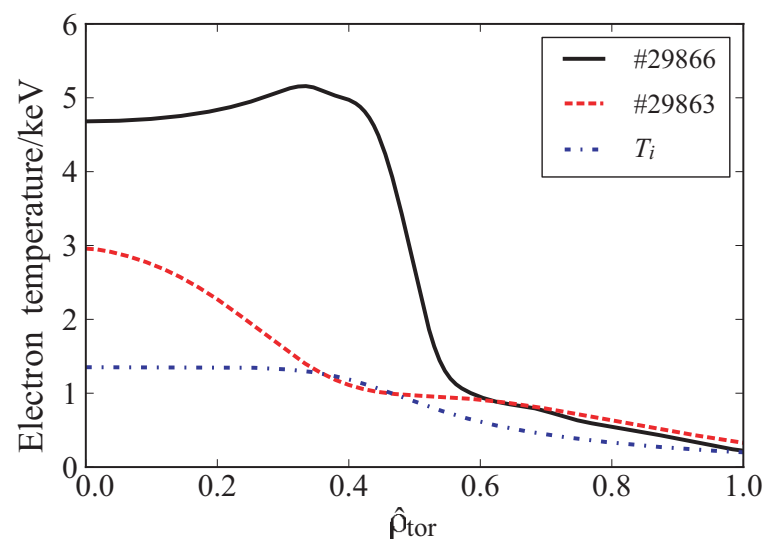

Figure 7. Comparison of the electron temperature profiles that were studied, and the ad hoc ion temperature profile used for both cases (blue, dashed).

with implications on the barrier strength: in discharge 29866, a counter-current perturbation had been used to create a large reversed-shear region, whereas in discharge 29863, a co-current perturbation lead to a monotonic $q$ profile. The experimental electron density and temperature profiles (see figure 7) from both discharges were used (along with reconstructed MHD equilibria from the CHEASE code [38]). The ion temperature in these discharges has not been measured except for its central value, which is used as a constraint for the ion temperature profile in the GENE simulation. For the present purpose, the effects of variations in the ion temperature profile can be regarded as strongly subdominant compared with changes in the electron temperature profile. We would also like to note that for these discharges, no pitch angle measurements were available to constrain the $q$ profile, and also the pressure profile has some freedom, given, e.g. the uncertainties in the ion pressure profile.

From earlier studies (see [39-41]), it is already wellknown that in such plasmas, the dominant microinstability at low wavenumbers is the TEM, although it has also been shown that the existence of a density barrier implies a co-existence 

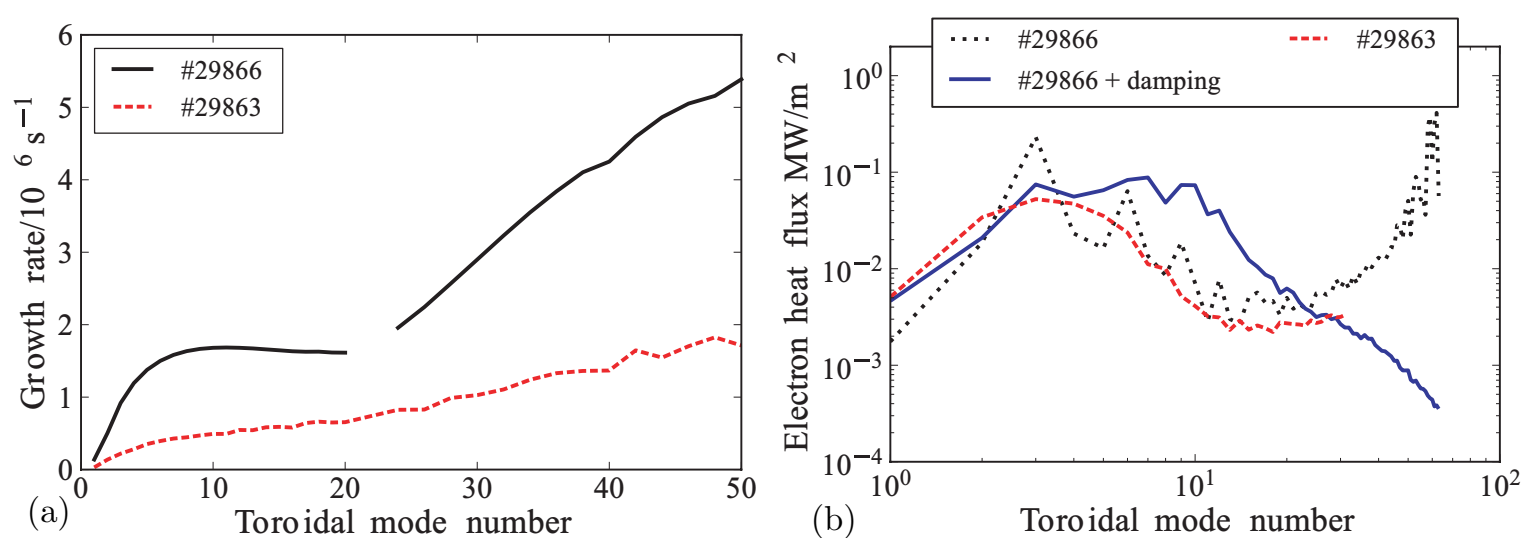

Figure 8. (a) Growth rates for both cases. ETGs are found unstable, whereas the low mode numbers are dominated by TEM, or even KBM for \#29866. The maximum mode number corresponds to $k_{\mathrm{y}} \rho_{\mathrm{s}}=5.7$ (\#29866) and 3.8 (\#29863). (b) Toroidal heat flux spectra for the studied cases. In the barrier case (black dotted), spectral blocking due to ETG modes occurs, which is countered by artificial damping to achieve converged large-scale simulations (blue solid line).

with ITG modes. For the nominal parameters in our study, we usually find dominant TEM instabilities, the steepest barrier case even surpassing the KBM threshold slightly. In addition to these instabilities on ion-gyroradius scale, however, both examined cases are also found to exhibit unstable ETG-driven modes-as evidenced by the strong increase of growth rates towards larger mode numbers (figure $8(a)$ ).

\subsection{Global GENE simulations for ITB discharges}

Here, we will restrict the nonlinear simulations to an electrostatic, collisionless treatment, deferring a closer examination of electromagnetic effects in this setting to a future publication. Linear parameter scans have found that the KBM can already be stabilized when relaxing the $T_{\mathrm{e}}$ barrier within the experimental uncertainty $(\Delta r \approx 1 \mathrm{~cm})$. Due to the disparate spatio-temporal scales of TEM and ETG turbulence, nonlinear simulations usually consider only one type of turbulence by setting up the domain size and resolution accordingly. From the growth rate spectra in figure $8(a)$, however, it is not obvious that there exists a suitable cutoff scale for such a separation. In fact, turbulence simulations for the barrier case (\#29866) exhibit energy accumulation at the smallest resolved scales, indicative of insufficiently resolved ETG activity. In order to achieve physically acceptable simulations of the TEMdriven turbulent transport (neglecting any coupling to ETGs), it is therefore necessary to introduce an artificial dissipation that affects only the smallest scales. Here, this is achieved by fourth-order hyperdiffusion terms which act on the radial and toroidal directions separately. Figure $8(b)$ demonstrates the effect of these terms on the toroidal heat flux spectra. The turbulence simulations are performed in a gradient-driven fashion, using adaptive Krook-type heat [42] and particle sources in order to maintain the initial profiles. With this approach, radial electron heat flux profiles as shown in figure 9 are obtained. In the discharge without a barrier, the obtained electron heat transport levels $(\sim 1.5 \mathrm{MW})$ are close to the experimental input power, which is $2.25 \mathrm{MW}$ in both cases. On the other hand, the simulated heat flux for the barrier discharge develops a strong peak in the barrier region, which at $\sim 18 \mathrm{MW}$ significantly exceeds the experimental value.

In order to assess the transport contribution driven by small-scale ETG modes, a second set of simulations was

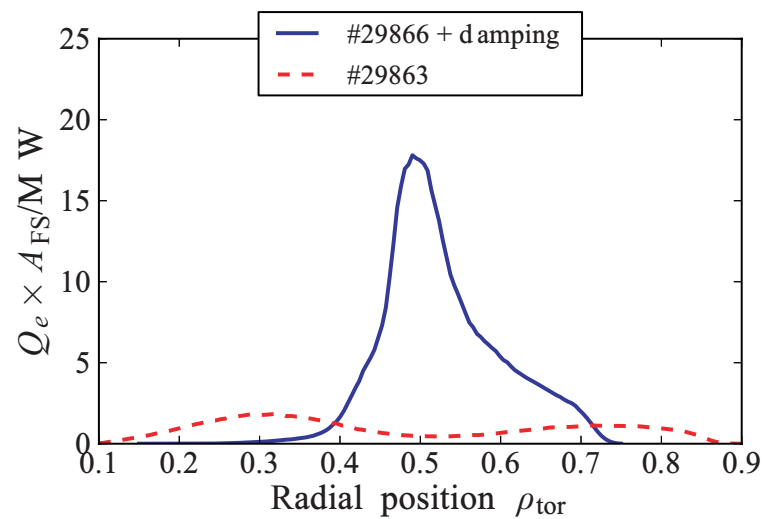

Figure 9. Radial profiles of electron heat transport rate (heat flux times flux-surface area), for the barrier case (blue solid) and the monotonic- $q$ case (red dashed).

performed for the barrier case, in which the simulation domain was restricted to the region of steepest temperature gradient and only scales relevant to ETG-type turbulence were considered. This set of simulations was carried out employing the adiabatic ion approximation (see, e.g., [5]), in which shielding effects due to impurities and different $T_{\mathrm{e}} / T_{\mathrm{i}}$ ratios can be retained as a combined parameter $\tau=Z_{\text {eff }} T_{\mathrm{e}} / T_{\mathrm{i}}$. The results of this scan are depicted in figure 10. For the whole range of $\tau$ values examined-ranging from a pure deuterium plasma with equal ion and electron temperatures to a more realistic setup with $\tau \sim 6-10$, the obtained electron heat flux values are comparable to the TEM-driven contribution. Considering the fact that both contributions are-for nominal input parameters significantly above the experimental flux, further parameter studies are required. As it turns out, relaxing the electron temperature profile within the experimental error bars (i.e. shifting the upper part of the barrier by $\Delta r \sim 1 \mathrm{~cm}$ ) reduces both the TEM and the ETG fluxes in that region by a factor 2. In either case, roughly $40 \%$ of the electron heat flux is generated by small-scale ETG turbulence.

With the present simulations, it is not possible to judge whether it is a valid approach in this case to introduce an artificial separation between the ion and electron scales. In fact, in an earlier work [43] examining a similar situation with both unstable TEM and ETG modes in the framework 


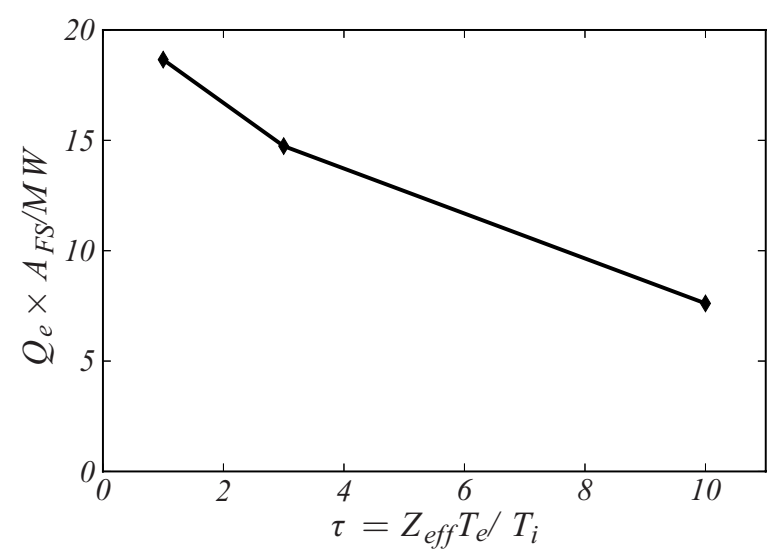

Figure 10. ETG-driven heat transport rate, varying the combined parameter $\tau=Z_{\text {eff }} T_{\mathrm{e}} / T_{\mathrm{i}}$.

of a multi-scale simulation (using a reduced mass ratio of $\left.m_{\mathrm{i}} / m_{\mathrm{e}}=400\right)$, a smooth coupling between ion and electrongyroradius scales was revealed. Further investigations along those lines will therefore be required to verify the fidelity of the present simulation results. In addition, electromagnetic effects neglected here may provide further stabilization in the barrier region, due to the interplay between an equilibrium pressure gradient contribution to the curvature drift [6], and compressional magnetic fluctuations.

\section{Conclusions}

In this paper, we have addressed a number of interrelated physics questions regarding high-performance discharges in view of ITER.

Finite-size effects across a range of tokamaks were examined by means of global gyrokinetic simulations retaining kinetic electrons, magnetic fluctuations, collisions and realistic geometry. Similar to the experience from simplified global models, the local approximation was found to be valid already for medium-sized tokamaks $\left(1 / \rho_{*} \sim 300\right)$, and good agreement with experimental transport levels was achieved. This suggests that predictive simulations of anomalous transport in the core of ITER plasmas can be performed by a coupling of flux-tube simulations via a transport solver, considerably reducing the overall computational effort.

Given that good plasma performance can only be achieved at high $\beta$, the character of turbulent transport in this regime has been investigated. It was shown that the simulation results suggest that experiments operating sufficiently close to the ideal MHD ballooning limit should be subject to a gradual shift from purely electrostatic to mixed electrostatic/electromagnetic turbulent transport, catalysed by MTMs which are either linearly or nonlinearly driven. Under which circumstances and to which degree this effect is reflected in the overall $\beta$ scaling of the energy confinement time remains to be studied in more detail. Clearly, the dependence of the quality of magnetic confinement on $\beta$ remains a crucial question to be addressed in light of ITER.

Another important issue is the threshold and stiffness of ITG-driven transport. In recent JET discharges, it had been observed that an increase in rotation led to reduced stiffness of the ion heat transport, which was not reproducible in gyrokinetic simulations. A comprehensive local parameter study using GENE was performed to find mechanisms that could be responsible for this reduction. In the simulations, it is confirmed that toroidal rotation alone (even in combination with low magnetic shear) cannot explain the observed ion temperature profile stiffness reduction. In addition, the usually assumed upshift of the critical gradient is counteracted (and often annihilated) by the PVG instability — an effect which is generally neglected in transport analyses. The main influence on stiffness reduction is due to finite $\beta_{\mathrm{e}}$ stabilization of ITG modes, in particular nonlinearly. Although this effect is still not sufficient to explain the small experimental stiffness, the obtained results stress the necessity of considering these effects in reduced transport models.

Finally, global simulations were carried out to analyze the transport mechanisms active in an electron-ITB. As an example for such a case, a TCV discharge was selected, along with a corresponding discharge without such a barrier. In the barrier case, the electron heat flux contributions due to trapped electron modes and ETG modes were found to be roughly equal, underscoring the importance of multi-scale treatments for such plasmas.

\section{Acknowledgments}

This work was carried out using the HELIOS supercomputer system at the Computational Simulation Centre of International Fusion Energy Research Centre (IFERC-CSC), Aomori, Japan, under the Broader Approach collaboration between Euratom and Japan, implemented by Fusion for Energy and JAEA. Further resources being employed were kindly provided on HPC-FF at the FZ Jülich, Germany, and the HPC facilities at RZG, Germany.

\section{References}

[1] Doyle E.J. et al 2007 Nucl. Fusion 47 S18

[2] Brizard A.J. and Hahm T.S. 2007 Rev. Mod. Phys. 79421

[3] Garbet X. et al 2010 Nucl. Fusion 50043002

[4] Jenko F. and the GENE Development Team 2013 The GENE code http://gene.rzg.mpg.de

[5] Jenko F., Dorland W., Kotschenreuther M. and Rogers B.N. 2000 Phys. Plasmas 71904

[6] Görler T., Lapillonne X., Brunner S., Dannert T., Jenko F., Merz F. and Told D. 2011 J. Comput. Phys. 2307053

[7] Lin Z., Ethier S., Hahm T.S. and Tang W.M. 2002 Phys. Rev. Lett. 88195004

[8] Candy J., Waltz R.E. and Dorland W. 2004 Phys. Plasmas $11 \mathrm{~L} 25$

[9] McMillan B.F., Lapillonne X., Brunner S., Villard L., Jolliet S., Bottino A., Görler T. and Jenko F. 2010 Phys. Rev. Lett. 105155001

[10] Barnes M., Abel I.G., Dorland W., Görler T., Hammett G.W. and Jenko F. 2010 Phys. Plasmas 17056109

[11] Petty C.C. 2008 Phys. Plasmas 15080501

[12] McDonald D.C. et al 2004 Plasma Phys. Control. Fusion 46 A 215

[13] Petty C.C. et al 2004 Phys. Plasmas 112514

[14] Dimits A.M. et al 2000 Phys. Plasmas 7969

[15] Parker S.E., Chen Y., Wan W., Cohen B.I. and Nevins W.M. 2004 Phys. Plasmas 112594

[16] Candy J. 2005 Phys. Plasmas 12072307

[17] Pueschel M.J., Kammerer M. and Jenko F. 2008 Phys. Plasmas 15102310

[18] Pueschel M.J. and Jenko F. 2010 Phys. Plasmas 17062307 
[19] Nevins W.M., Wang E. and Candy J. 2011 Phys. Rev. Lett. 106065003

[20] Wang E., Nevins W.M., Candy J., Hatch D.R., Terry P.W. and Guttenfelder W. 2011 Phys. Plasmas 18056111

[21] Hatch D.R., Pueschel M.J., Jenko F., Nevins W.M., Terry P.W. and Doerk H. 2012 Phys. Rev. Lett. 108235002

[22] Pueschel M.J., Terry P.W., Jenko F., Hatch D.R., Nevins W.M., Görler T. and Told D. 2013 Phys. Rev. Lett. 110155005

[23] Hatch D.R., Pueschel M.J., Jenko F., Nevins W.M., Terry P.W. and Doerk H. 2013 Phys. Plasmas 20012307

[24] Applegate D.J., Roach C.M., Connor J.W., Cowley S.C., Dorland W., Hastie R.J. and Joiner N. 2007 Plasma Phys. Control. Fusion 491113

[25] Vermare L., Angioni C., Bottino A., Peeters A.G. and the ASDEX Upgrade Team 2008 J. Phys. Conf. Ser. 123012040

[26] Told D., Jenko F., Xanthopoulos P., Horton L.D., Wolfrum E. and the ASDEX Upgrade Team 2008 Phys. Plasmas 15102306

[27] Doerk H., Jenko F., Pueschel M.J. and Hatch D.R. 2011 Phys. Rev. Lett. 106155003

[28] Doerk H., Jenko F., Görler T., Told D., Pueschel M.J. and Hatch D.R. 2012 Phys. Plasmas 19055907

[29] Dickinson D., Roach C.M., Saarelma S., Scannell R., Kirk A. and Wilson H.R. 2012 Phys. Rev. Lett. 108135002

[30] Mantica P. et al 2009 Phys. Rev. Lett. 102175002

[31] Mantica P. et al 2011 Phys. Rev. Lett. 107135004
[32] Artaud J.F. et al 2010 Nucl. Fusion 50043001

[33] Lapillonne X. et al 2009 Phys. Plasmas 16032308

[34] Weiland J. and Hirose A. 1992 Nucl. Fusion 32151

[35] Houlberg W.A., Shaing K.C., Hirshman S.P. and Zarnstroff M.C. 1997 Phys. Plasmas 43230

[36] Sauter O., Coda S., Goodman T.P., Henderson M.A., Behn R. Bottino A., Fable E., Martynov A., Nikkola P. and Zucca C. 2005 Phys. Rev. Lett. 94105002

[37] Zucca C., Sauter O., Asp E., Coda S., Fable E., Goodman T.P and Henderson M.A. 2009 Plasma Phys. Control. Fusion 51015002

[38] Lütjens H., Bondeson A. and Sauter O. 1996 Comput. Phys. Commun. 97219

[39] Bottino A., Sauter O., Camenen Y. and Fable E. 2006 Plasma Phys. Control. Fusion $\mathbf{4 8} 215$

[40] Fable E., Sauter O., Coda S., Goodman T.P., Henderson M.A., Weisen H., Zabolotsky A., Zucca C. and the TCV Team 2006 Plasma Phys. Control. Fusion 481271

[41] Lapillonne X., Brunner S., Sauter O., Villard L., Fable E., Görler T., Jenko F. and Merz F. 2011 Plasma Phys. Control. Fusion 53054011

[42] Lapillonne X., McMillan B.F., Görler T., Brunner S., Dannert T., Jenko F., Merz F. and Villard L. 2010 Phys. Plasmas 17112321

[43] Görler T. and Jenko F. 2008 Phys. Rev. Lett. 100185002 